\title{
Clinical-therapeutic management of drooling: Review and update
}

\author{
Javier Silvestre-Rangil ${ }^{1}$, Francisco-Javier Silvestre ${ }^{2}$, Angel Puente-Sandoval ${ }^{1}$, Juan Requeni-Bernal ${ }^{1}$, Juan- \\ Manuel Simo-Ruiz ${ }^{3}$
}

${ }^{1}$ Collaborating dental surgeon of the Stomatology Unit of Dr. Peset University Hospital

${ }^{2}$ Assistant Professor of the Department of Stomatology of the University of Valencia. Head of the Stomatology Unit of Dr. Peset University Hospital

${ }^{3}$ Dental surgeon of the Stomatology Unit of Dr. Peset University Hospital. Valencia (Spain)

Correspondence:

Hospital Universitario Dr. Peset

Consultas Externas

C/ Juan de Garay s/n

46017 - Valencia (Spain)

francisco.silvestre@uv.es

Received: $21 / 08 / 2010$

Accepted: $14 / 11 / 2010$

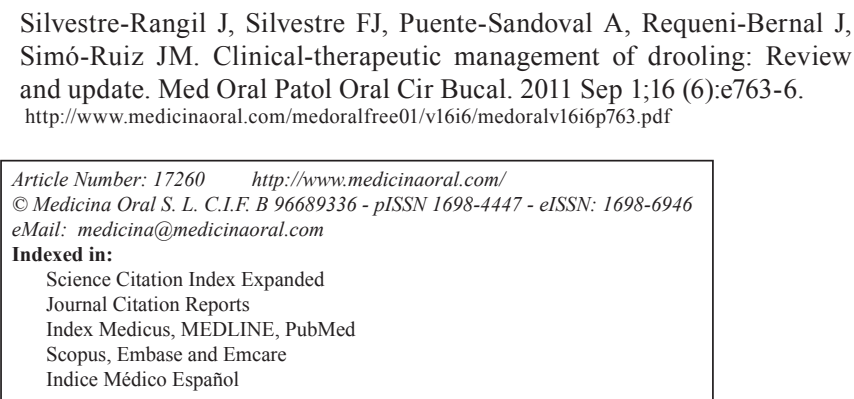

Silvestre-Rangil J, Silvestre FJ, Puente-Sandoval A, Requeni-Bernal J, Simó-Ruiz JM. Clinical-therapeutic management of drooling: Review and update. Med Oral Patol Oral Cir Bucal. 2011 Sep 1;16 (6):e763-6. http://www.medicinaoral.com/medoralfree01/v16i6/medoralv16i6p763.pdf

\begin{abstract}
Drooling is the uncontrolled leakage of saliva outside the mouth, generally as a result of difficulty in swallowing the saliva produced. Many factors contribute to drooling, though it is more commonly seen in children with brain paralysis - particularly those receiving anticonvulsivant medication. Drooling is also often seen in patients with lip sealing problems or malocclusions such as anterior open bite.

Clinically, the affected patients can develop skin irritation or abrasions, problems of hygiene, unpleasant smell and - in the more severe presentations - the need to wear protectors or frequently change clothing.

Treatment of this disorder is complex, and should be addressed from a multidisciplinary perspective, with planning on an individualized basis. Among the different existing managements, myofunctional therapy, behavioral change programs and drug treatments are the most widely used options, though there are also more invasive surgical techniques designed to reduce or cause submandibular saliva secretion to be rerouted towards posterior zones of the oral cavity. In any case, no scientific evidence-based management protocol has yet been established capable of affording favorable results in the majority of cases.

The present study offers a review and update on the clinical and dental management aspects of drooling.
\end{abstract}

\section{Key words: Drooling, clinical management, treatment of drooling.}

\section{Introduction}

Drooling is the uncontrolled leakage of saliva outside the mouth, and may be attributed to a number of causes. In small children drooling is normal until 18-24 months of age, though in some cases the condition can persist up to four years of age, and certain upper airway infections moreover may exacerbate the problem. However, as the child grows older, these problems tend to disappear (1). Drooling can also be observed in patients with certain neurodegenerative diseases, though it is usually seen in patients with developmental disorders - particularly those characterized by alterations in orofacial neuromuscular control (2).

Drooling adversely affects patient quality of life and can 
cause social rejection problems. These are individuals that require constant care due to their problems of hygiene, unpleasant smell, skin irritations and abrasions, increased susceptibility to perioral infections and - depending on the severity of the condition - a certain degree of dehydration. Constant changing of clothes may prove necessary in the most severe presentations (3). Different studies point to alterations in oral mechanisms and functions, such as lip or oral sealing problems, as further causes of drooling, along with swallowing difficulties. Drooling is not a result of hypersialia or sialorrhea characterized by increased saliva production; indeed, in some cases drooling is observed in the presence of limited saliva output. Rather, the problem is due to saliva swallowing difficulty. This and the favoring influence of a series of factors give rise to continuous saliva leakage outside the mouth (4).

Saliva is produced by the major and minor salivary glands, though most of the production corresponds to the former. The major salivary glands comprise three pairs of glands symmetrically distributed on either side of the face. The parotid glands produce more watery serosal saliva, fundamentally as a result of stimulation during meals. The submandibular and sublingual glands in turn produce more mucinous, viscous saliva that is secreted in a more constant manner throughout the day (5). This is the type of saliva found in greater proportion in drooling.

The present study offers a review and update on this important problem in the clinical and dental management of special patients, and particularly of disabled individuals.

\section{Search Methodology}

A Medline/PubMed search was carried out, identifying the articles that describe different hypotheses relating to the clinical and therapeutic management of drooling, based on the following key words: drooling AND management AND treatment $(n=210)$. We selected those articles (without publication date restrictions) containing some key word in the title - including clinical trials of adequate sample size or exhaustive reviews of drooling in English. Based on the established screening criteria, a total of 22 articles were considered.

\section{Review of the literature}

\section{-Physiopathology of drooling and etiological factors}

A correct swallowing reflex is essential for the swallowing of saliva. This complex basic function is mediated by orofacial neuromuscular systems and involves a series of reflex sequential and coordinated movements of the mandible, lips, tongue, and pharyngeal, laryngeal and esophageal muscles. This motor sequence in turn is coordinated by a swallowing center located in the spinal cord. Swallowing comprises three phases: oral, pharyngeal and esophageal (6).
The clinical causes of drooling include neurological alterations on one hand and local buccodental problems on the other. Among the neurological causes, brain paralysis is the most common. In effect, $58 \%$ of all children with brain paralysis suffer drooling, and in 33\% of the cases the condition is severe (7). Other neurological conditions associated with drooling are amyotrophic lateral sclerosis (ALS), Parkinson's disease (8), cerebrovascular accidents (e.g., stroke), patients with important paralysis, congenital suprabulbar paralysis, certain cases of encephalitis, hypoxic encephalopathy, severe mental retardation, hydrocephalus and certain rare syndromes such as Moebius syndrome, Angleman syndrome, Freeman Sheldon syndrome, or Landau-Kleffner syndrome (7).

Local causes of drooling in turn include problems leading to an open mouth position, a lack of lip sealing, certain malocclusions, atrophy or important reabsorption of the anterior alveolar crest, certain tongue deformities, and anesthesia or hypoesthesia of the anterior sectors of the mouth (9).

Other important factors that favor drooling are an inadequate body posture, though particularly of the head, which must remain erect in order to prevent drooling. A lack of sensitivity in the oral phase of swallowing can also be a causal factor, in the same way as certain cranial nerve lesions (facial nerve (pair VII) and hypoglossal nerve (pair XII)) and alterations precluding mandibular stabilization - the latter being a prior requirement for correct swallowing. In disabled patients with brain paralysis or mental retardation, emotional state and the degree of concentration are also influencing factors (2), and these subjects moreover are at a high risk of developing dental caries (10).

Drooling can coexist with other medical conditions, though most affected patients are children or young individuals with mental disabilities and/or neurological problems (9). A clinical condition commonly associated with drooling is epilepsy. Other associated factors are allergic rhinitis, upper airway disorders, and gastroesophageal reflux. Although sialorrhea is not a cause of drooling, it can exacerbate the problem, with an increase in salivary flow under resting conditions, and it may be a side effect of certain drug treatments $(9,11)$. A number of methods have been described for the clinical evaluation of drooling. In 1982, Sochaniwskyj (12) described a technique based on collection with a chin cup of the saliva leaking from the mouth over a period of 30 minutes. This was repeated five times in order to calculate an average and thus avoid variability of the data obtained. The technique did not involve the collection of whole saliva, but only the saliva leaking through the lips and reaching the chin.

In 1988, Thomas-Storell and Greenberg (13) developed a classification for measuring the intensity or grade of 
drooling. Five grades were considered: $1=$ dry lips, with no drooling; 2 = constantly humid lips; 3 = lip humidity extending to the chin (moderate drooling); $4=$ humidity reaching the clothing around the neck region (severe drooling); and $5=$ humidity or wetness affecting the clothes, hands and objects (profuse drooling).

-Therapeutic options in relation to drooling

When considering the clinical management of drooling, and on attempting to design intervention strategies to improve and correct the neuromuscular imbalances of the patient, it must be taken into account that this is a complex subject which moreover requires a multidisciplinary approach involving dentists, pediatricians, surgeons, physiotherapists, specialists in logopedics, etc. On the other hand, there is no single protocol that can be applied to the different clinical cases seen; rather, an individualized management strategy must be developed for each patient (14).

The different therapeutic options found in the literature include myofunctional therapy, behavioral change programs, drug treatments and surgery designed to correct or avoid drooling $(7,14-16)$. Other options have not been considered in this study, due to their side effects (as in the case of radiotherapy) or because of their minority application in current clinical practice (as in the case of acupuncture) (17).

Myofunctional therapy is physiotherapy designed to rehabilitate orofacial neuromuscular function from early patient ages, with improvements in nasal breathing, lip sealing and oral closure. Such techniques seek to ensure adequate control of neuromuscular groups in order to improve chewing and swallowing, facilitate correct feeding via the oral route, and secure adequate control of the position of the head (9).

Myofunctional therapy comprises a series of techniques and procedures that improve or correct the abovementioned functions, help reduce negative habits and improve patient aesthetics.

The programs are to be established on an individualized basis and comprise repetitive and coordinated physical exercises; lip, mandible and throat pressure techniques; and vibratory stimulation for about two minutes over these anatomical locations. There are also orthopedic techniques involving the use of intraoral acrylic plates to stimulate lingual retrusion or the lips, for example, according to the method developed by Castillo-Morales (18).

While logopedic intervention can suffice to ensure adequate saliva control, patient mental retardation or verbal or nonverbal comprehension difficulties unfortunately often complicate the application of such techniques (1). Another management option is the so-called feedback technique (9), based on the monitorization of the muscle group targeted for stimulation using electromyography (EMG). Two adhesive surface electrodes are placed on over the muscle from which feedback is to be obtained. When the muscle contracts, the electromyograph informs of the change in activity by means of an acoustic or luminous signal. In this way the patient is aware of the activity, e.g., swallowing, and may thus consciously correct or improve certain components of swallowing function. Such EMG-mediated acoustic feedback can have a positive impact on patient training and on the improvement of oral motor function.

Behavioral change programs have been used, based on reinforcement techniques designed to increase or decrease certain previously determined behaviors (19). A first measure is to teach the required behavior, such as correct swallowing, and then to reinforce such behavior when it is correctly executed. Such programs can be used in combination with the acoustic feedback technique to reduce drooling, conditioning the patient to swallow each time a signal is heard from the electronic system equipped with a timer device. It is also possible to use a chin humidity sensor that triggers a signal when humidity increases - thereby inducing the patient to swallow. These are some examples of anti-drooling techniques which nevertheless can have certain inconveniences, such as the complication of social relations while the system is being used. Moreover, while acceptable results have been obtained over the short term, the beneficial effects are seen to fade over longer periods of time (19).

Another treatment option is represented by drug therapy designed to reduce salivary flow. The salivary glands are controlled by the autonomic nervous system; in this sense, muscarinic cholinergic receptor block induces an important decrease in salivary flow, and anticholinergic drugs have therefore been used to control drooling. However, such drug substances can induce important undesirable effects such as vomiting, diarrhea, irritability, mood changes and insomnia. Those described in the literature for the treatment of drooling have been atropine sulfate, glycopyrrolate and scopolamine.

Mier et al. (20) carried out a double-blind study of 39 children with drooling administered either glycopyrrolate or placebo. The results showed considerable improvement with the active drug. Scopolamine likewise has been used in transdermal patch form, offering longer action and a low incidence of side effects, though it must be avoided in patients with cardiac and gastrointestinal disorders.

On the other hand, botulinum toxin has been used in patients with bruxism, tremor, spasticity, rigidity or muscle dystonia, and has also been proposed in application to drooling. It exerts a local and reversible effect, without the risk of side effects, and can be used in certain cases of drooling characterized by a predominant spasticity component (21).

As a last treatment resort, surgery has been proposed to 
reduce salivary secretion, rather than to improve saliva transit in the mouth $(22,23)$. Techniques as radical as salivary gland resection or salivary duct ligation have been proposed, though salivary duct transpositioning has been the most widely used option and involves fewer adverse effects. Surgical treatment of drooling generated considerable controversy, since it often resulted in extreme dry mouth, loss of taste sensation, tongue mobility problems in the anterior sector, swelling and a tendency towards important sialoadenitis. Submandibular gland duct transpositioning towards the tonsillar pillars, designed to facilitate the swallowing of saliva, has also given rise to postoperative problems such as the appearance of ranulas or loss of smooth muscle function of the terminal sphincters. In any case, careful evaluation is required of the possible treatment alternatives in each individual patient before considering the surgical techniques.

\section{-Clinical-dental management of drooling}

Drooling is frequent in patients requiring special care, particularly in those who are mentally disabled, and has a strong impact upon quality of life (24).

A good diagnosis of the problem must be established, with identification of the implicated factors in each case. This is to be followed by an individualized treatment plan that should be as little invasive as possible.

The functional swallowing problems should be dealt with in early stages, based on physiotherapy and functional re-education measures involving repetitive exercises to favor correct swallowing (9).

In disabled patients (e.g., with cerebral palsy), myofunctional therapy should be started as soon as possible. Attempts likewise should be made to avoid or correct anterior open bite or other vertical malocclusions, and to seek correct lip sealing through oral closure stimulation techniques.

When these treatment measures prove ineffective, certain antisecretory drugs can be used to reduce salivary flow. In this context, the most widely used drugs are atropinic, anticholinergic and antihistaminic agents. On the other hand, surgery should be avoided as far as possible, since it is only able to afford palliative effects.

\section{References}

References with links to Crossref - DOI

1. Senner JE, Logemann J, Zecker S, Gaebler-Spira D. Drooling, saliva production, and swallowing in cerebral palsy. Dev Med Child Neurol. 2004;46:801-6.

2. Lespargot A, Langevin MF, Muller S, Guillemont S. Swallowing disturbances associated with drooling in cerebral-palsied children. Dev Med Child Neurol. 1993;35:298-304.

3. Blasco PA, Allaire JH. Drooling in the developmentally disabled: management practices and recommendations. Consortium on Drooling. Dev Med Child Neurol. 1992;34:849-62.

4. Tahmassebi JF, Curzon ME. The cause of drooling in children with cerebral palsy -- hypersalivation or swallowing defect? Int J Paediatr Dent. 2003;13:106-11.

5. Erasmus CE, Van Hulst K, Rotteveel LJ, Jongerius PH, Van Den Hoogen FJ, Roeleveld N, et al. Drooling in cerebral palsy: hypersali- vation or dysfunctional oral motor control? Dev Med Child Neurol. 2009;51:454-9.

6. Arvedson J, Clark H, Lazarus C, Schooling T, Frymark T. The effects of oral-motor exercises on swallowing in children: an evidencebased systematic review. Dev Med Child Neurol. 2010;52:1000-13.

7. Meningaud JP, Pitak-Arnnop P, Chikhani L, Bertrand JC. Drooling of saliva: a review of the etiology and management options. Oral Surg Oral Med Oral Pathol Oral Radiol Endod. 2006;101:48-57.

8. Merello M. Sialorrhoea and drooling in patients with Parkinson's disease: epidemiology and management. Drugs Aging. 2008;25:100719.

9. Morales Chávez MC, Nualart Grollmus ZC, Silvestre-Donat FJ. Clinical prevalence of drooling in infant cerebral palsy. Med Oral Patol Oral Cir Bucal. 2008;13:E22-6.

10. Rodrigues dos Santos MT, Masiero D, Novo NF, Simionato MR. Oral conditions in children with cerebral palsy. J Dent Child (Chic). 2003;70:40-6.

11. Tahmassebi JF, Curzon ME. Prevalence of drooling in children with cerebral palsy attending special schools. Dev Med Child Neurol. 2003;45:613-7.

12. Sochaniwskyj AE. Drool quantification: noninvasive technique. Arch Phys Med Rehabil. 1982;63:605-7.

13. Thomas-Stonell N, Greenberg J. Three treatment approaches and clinical factors in the reduction of drooling. Dysphagia. 1988;3:73-8.

14. Lloyd Faulconbridge RV, Tranter RM, Moffat V, Green E. Review of management of drooling problems in neurologically impaired children: a review of methods and results over 6 years at Chailey Heritage Clinical Services. Clin Otolaryngol Allied Sci. 2001;26:76-81.

15. Nunn JH. Drooling: review of the literature and proposals for management. J Oral Rehabil. 2000;27:735-43.

16. Yam WK, Yang HL, Abdullah V, Chan CY. Management of drooling for children with neurological problems in Hong Kong. Brain Dev. 2006;28:24-9.

17. Wong V, Sun JG, Wong W. Traditional Chinese medicine (tongue acupuncture) in children with drooling problems. Pediatr Neurol. 2001;25:47-54.

18. Limbrock GJ, Hoyer H, Scheying H. Drooling, chewing and swallowing dysfunctions in children with cerebral palsy: treatment according to Castillo-Morales. ASDC J Dent Child. 1990;57:445-51. 19. Fairhurst CB, Cockerill H. Management of drooling in children. Arch Dis Child Educ Pract Ed. 2011;96:25-30.

20. Mier RJ, Bachrach SJ, Lakin RC, Barker T, Childs J, Moran M. Treatment of sialorrhea with glycopyrrolate: A double-blind, doseranging study. Arch Pediatr Adolesc Med. 2000;154:1214-8.

21. Erasmus CE, Van Hulst K, Van Den Hoogen FJ, Van Limbeek J, Roeleveld N, Veerman EC, et al. Thickened saliva after effective management of drooling with botulinum toxin A. Dev Med Child Neurol. 2010;52:e114-8.

22. Reed J, Mans CK, Brietzke SE. Surgical management of drooling: a meta-analysis. Arch Otolaryngol Head Neck Surg. 2009;135:92431 .

23. Osorio A, Moreira-Pinto J, Oliveira L, Ferreira-de-Sousa JA, Cidade-Rodrigues JA. Bilateral submandibulectomy for the treatment of drooling in children with neurological disability. Eur J Pediatr Surg. 2009;19:377-9

24. Scully C, Limeres J, Gleeson M, Tomás I, Diz P. Drooling. J Oral Pathol Med. 2009;38:321-7. 